\title{
Solar Transparent Radiators by Optical Nanoantennas
}

Gustav J önsson, Daniel Tordera, Tavakol Pakizeh, Manoj J aysankar, Vladimir Miljkovic, Lianming Tong, Magnus J onsson and Alexandre Dmitriev

The self-archived postprint version of this journal article is available at Linköping University Institutional Repository (DiVA):

http:/ / urn.kb.se/ resolve?urn=urn:nbn:se:liu:diva- 143684

N.B.: When citing this work, cite the original publication.

J önsson, G., Tordera, D., Pakizeh, T., J aysankar, M., Miljkovic, V., Tong, L., J onsson, M., Dmitriev, A., (2017), Solar Transparent Radiators by Optical Nanoantennas, Nano letters (Print), 17(11), 6766-6772. https:// doi.org/ 10.1021/ acs.nanolett.7b02962

Original publication available at:

https:/ / doi.org/ 10.1021/ acs.nanolett.7b02962

Copyright: American Chemical Society

http:// pubs.acs.org/ 


\section{Solar transparent radiators by optical nanoantennas}

Gustav E. Jönsson ${ }^{1}$, Daniel Tordera ${ }^{2, \dagger}$, Tavakol Pakizeh ${ }^{6}$, Manoj Jaysankar ${ }^{1}$, Vladimir Miljkovic $^{4}$, Lianming Tong ${ }^{5}$, Magnus P. Jonsson ${ }^{2}$ and Alexandre Dmitriev ${ }^{* 1,3,7}$

${ }^{1}$ Department of Physics, Chalmers University of Technology, 41296 Gothenburg, Sweden.

${ }^{2}$ Laboratory of Organic Electronics, Linköping University, SE-601 74 Norrköping, Sweden.

${ }^{3}$ Department of Physics, University of Gothenburg, Gothenburg 412 96, Sweden

${ }^{4}$ NILT Sweden Filial, Stena Center 1B, 41292 Gothenburg, Sweden.

${ }^{5}$ Institute of Physics, Chinese Academy of Sciences, 100190 Beijing, P. R. China.

${ }^{6}$ Faculty of Electrical Engineering, K. N. Toosi University of Technology, Tehran 1631714191, Iran.

${ }^{7}$ Geballe Laboratory for Advanced Materials, Stanford University, Stanford, California 943054045, USA

† Present address: Holst Centre/TNO, High Tech Campus 31, 5605 KN Eindhoven, The Netherlands 


\begin{abstract}
Architectural windows are a major cause of thermal discomfort as the inner glazing during cold days can be several degrees colder than the indoor air. Mitigating this, the indoor temperature has to be increased, leading to unavoidable thermal losses. Here we present solar thermal surfaces based on complex nanoplasmonic antennas that can raise the temperature of window glazing by up to $8 \mathrm{~K}$ upon solar irradiation while transmitting light with a color rendering index of 98.76. The nanoantennas are directional, can be tuned to absorb in different spectral ranges and possess a structural integrity that is not substrate dependent, and thus they open up for application on a broad range of surfaces.
\end{abstract}

Keywords: thermoplasmonics, thermal management, plasmonic heating, optical antennas, plasmonic nanostructures. 
A sustainable production and use of energy is one of the biggest challenges faced by the modern society. New ways to improve thermal energy management are therefore crucial. ${ }^{1-3}$ The indoorsoutdoors thermal balance plays an important role in the thermal energy efficiency of buildings and living spaces. At interior temperature maintained at $20{ }^{\circ} \mathrm{C}$ the state-of-the-art low-emission windows have typical inner surface temperatures of $16^{\circ} \mathrm{C}$ and $14{ }^{\circ} \mathrm{C}$ for outdoor temperatures of $0{ }^{\circ} \mathrm{C}$ and $-10{ }^{\circ} \mathrm{C}$, respectively. ${ }^{4}$ The comfortable indoors climate is achieved when the mean radiant temperature (MRT, temperature of the walls, windows, ceiling and the floor) is close to the temperature of the indoor air. As a rule of thumb, for a change of $3{ }^{\circ} \mathrm{C}$ of the MRT, the air temperature needs to be adjusted by $2{ }^{\circ} \mathrm{C}$ in the opposite direction to balance the perception of a comfortable indoor environment. ${ }^{5}$ That is, elevating the windows' surface temperature by several degrees could allow a substantial lowering of the indoor temperature during the cold time of the year while maintaining a comfortable indoor climate and leading to considerable energy savings. Inversely, a cold non-absorbing glazing would be beneficial if the indoor climate is perceived to be too warm. Making the front and backside of a window to absorb solar radiation differently, the heat generator can turn into a solar deflector by reversing the window orientation.

Here we introduce the concept of a transparent and colorless solar-powered heater (radiator). Such a device could transform ordinary windows into radiators, utilizing plasmonic optical nanoantennas as heat harvesters. Nanoplasmonic materials are receiving great attention in the development of various conversion schemes of sunlight into electricity or the stimulation of various chemical and mechanical processes. ${ }^{6-14}$ Light-absorbing plasmonic materials utilize the strong coupling of electromagnetic radiation in the ultraviolet, visible or near infrared (UV-VISNIR) spectral range to the collective oscillations of electrons (plasmons). ${ }^{15}$ Plasmon-enabled absorbing surfaces typically comprise a collection of individual optical antennas that feature 
specifically configured localized surface plasmon resonances and collectively display spectrally and directionally tunable light absorption. Careful design of structure and materials composition enables controlled engineering of properties including spectral and directional selectivity and nanoplasmon decay pathways. ${ }^{16-18}$ Highly absorbing plasmonic metasurfaces are supported on an underlying substrate and can be realized as macroscopic functional layers. ${ }^{19-20}$

In this work, we present thermoplasmonic surfaces composed of arrays of multi-element (one and three) gold and nickel plasmonic nanoantennas on glass. The surfaces display a number of absorption and scattering resonances that correlates with the number of antenna elements. We present both the spectral absorption and the absolute temperature increase of these surfaces upon simulated solar illumination, showing an increase of up to $8 \mathrm{~K}$ for the optimum configuration. Interestingly, the systems provide directionality in light absorption, meaning that the absorption is different for light incident the opposite sides of such surface. This also transfers to directionality in heat generation. The plasmonic optical properties, heating profiles and directionality of the surfaces agree with combined optical and thermal simulations. The surfaces are transparent in the visible (about $75 \%$ of overall transmittance) and present very high color rendering index reaching 98.76. This makes them virtually colorless and suitable for applications where transparency is required. The nanoantenna surfaces could be tuned to absorb at different wavelengths (e.g. in the NIR), are substrate independent and mechanically robust.

We prepared thermoplasmonic nanoantenna surfaces using hole-mask colloidal lithography ${ }^{20}$ as described in the Experimental Section. In brief, we used $230 \mathrm{~nm}$ polystyrene beads to define hole-masks and thermally evaporated material stacks consisting of one or three layers of gold or nickel, and $\mathrm{SiO}_{2}$ (for gold) or $\mathrm{Al}_{2} \mathrm{O}_{3}$ (for nickel) dielectric layers on top of each metal layer (Figure 1a). We also made elliptical nanoantennas by evaporation at a $45^{\circ}$ angle (for the 1:1.4 
ratio between the axis). Figure 1b shows an SEM image of the Ni three-element elliptical plasmonic nanoantennas. The elements present a compact vertical arrangement of metallic nanoellipses of gradually decreasing size, spaced by dielectric layers. The nanoantennas have a characteristic centre-to-centre distance of around $630 \mathrm{~nm}$, as calculated from the radial distribution function (RDF) of SEM images. Figure 1c shows a photograph of a produced thermal glass (based on the nickel three-element nanoantennas) that is indeed largely transparent in the visible and with excellent color preservation, two major criteria to be met with glass windows. Below we also quantify the color rendering in transmission for such glasses, evidencing their essentially colorless appearance.

a
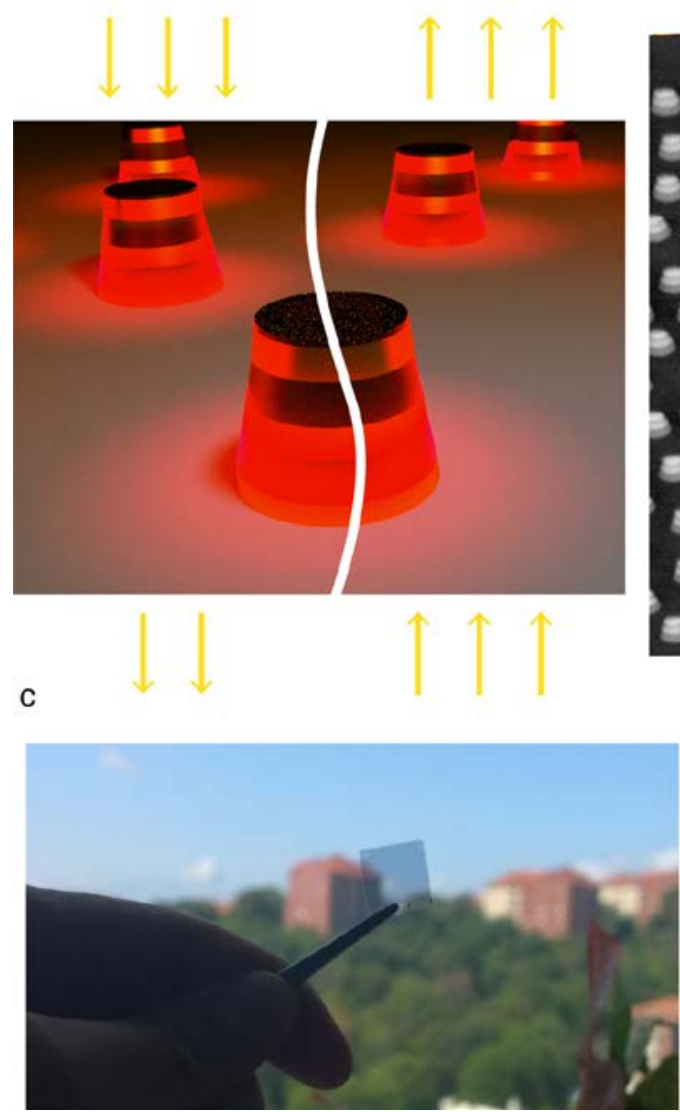

b
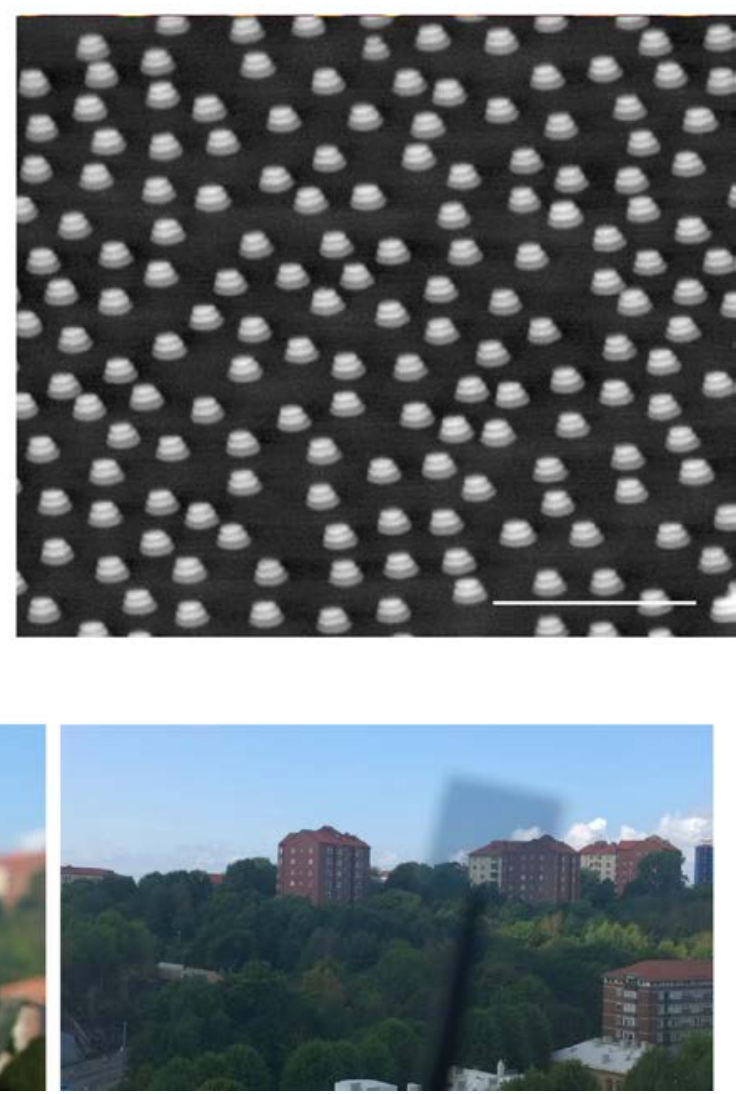
Figure 1. a) Artistic rendering of a three-element nanoantenna thermoplasmonic surface, illuminated from the front side (left) or back side (right). Point heat source nanoantennas collectively build up the thermal surface. Due to the directional solar light absorption, most of the nanoantenna surfaces are more efficient in generating heat when illuminated from top (air side). b) SEM micrograph of a representative thermal surface built on sparse arrays of the threeelement $\mathrm{Ni}-\mathrm{Al}_{2} \mathrm{O}_{3}$ elliptical nanoantennas (scale-bar $2 \mu \mathrm{m}$ ). c) Photographs of a glass slides with Ni three-element nanoantennas (see text for details of their geometry).

Next, we characterized the optical properties of the nanoantenna surfaces. Figure 2 shows the optical absorption, experimental (a-c) and simulated (d-f), of the different nanoantenna arrays (one- and three-element gold nanoantennas, and three-element elliptical nickel nanoantennas). The spectroscopic absorption was measured by using the integrating sphere (for experimental and simulations details - see Experimental Section below). We find a good match between experiments and simulations, including overall spectral behavior, peak positions, and directionality of light absorption. Single-element gold antennas (Figure 2a) have a distinct plasmonic peak at $1085 \mathrm{~nm}$. Adding more elements to the nanoantennas gives more spectrally rich absorption, with the three-element gold nanoantennas (separated by $40 \mathrm{~nm} \mathrm{SiO}_{2}$ spacers) providing absorption peaks at $687 \mathrm{~nm}, 866 \mathrm{~nm}$ and $1147 \mathrm{~nm}$ (Figure 2b). This is due to near-, mid- and far-field coupling of the nanodisks in the antennas. ${ }^{21}$ Indeed, numerically simulated near-field plots of the same system (Figures 2g-j) show that the peak at $823 \mathrm{~nm}$ arises from plasmonic coupling between the mid and top elements of the nanoantenna while the longer wavelength peak at $1050 \mathrm{~nm}$ corresponds to coupling between the lower and mid elements of the antenna. Both the overall absorption and the absorption at the peaks increased when adding more 
elements. The former is promoted by a spectral broadening of the absorption caused by additional plasmon modes for multi-elements nanoantennas, and the latter is due to spectral overlap of these modes. For nanoantennas with only one element the absorption has a slight directionality towards the substrate, which is related to the difference in refractive index of the substrate and the surrounding air. While a single nanodisk dipolar optical antenna in homogeneous medium is essentially bi-directional, it is well-known that the presence of a substrate substantially modifies the absorption/scattering balance and the electromagnetic nearfield distribution. ${ }^{22-23}$ Adding elements to nanoantennas results in significantly stronger (and opposite) directional light absorption. We attribute this to the compact sub-wavelength design and tight near-field coupling of all elements, where the near-field of the nanoantennas, as shown in our previous work, allows to efficiently beam light from local sources (e.g., quantum dots), featuring directionality in emission reaching $12-15 \mathrm{~dB} \cdot{ }^{21}$ Furthermore, light is more efficiently absorbed when incident from the frontside of the three-element nanoantenna surface and the effects of the substrate are made negligible by the intrinsic directionality of the nanoantenna. 

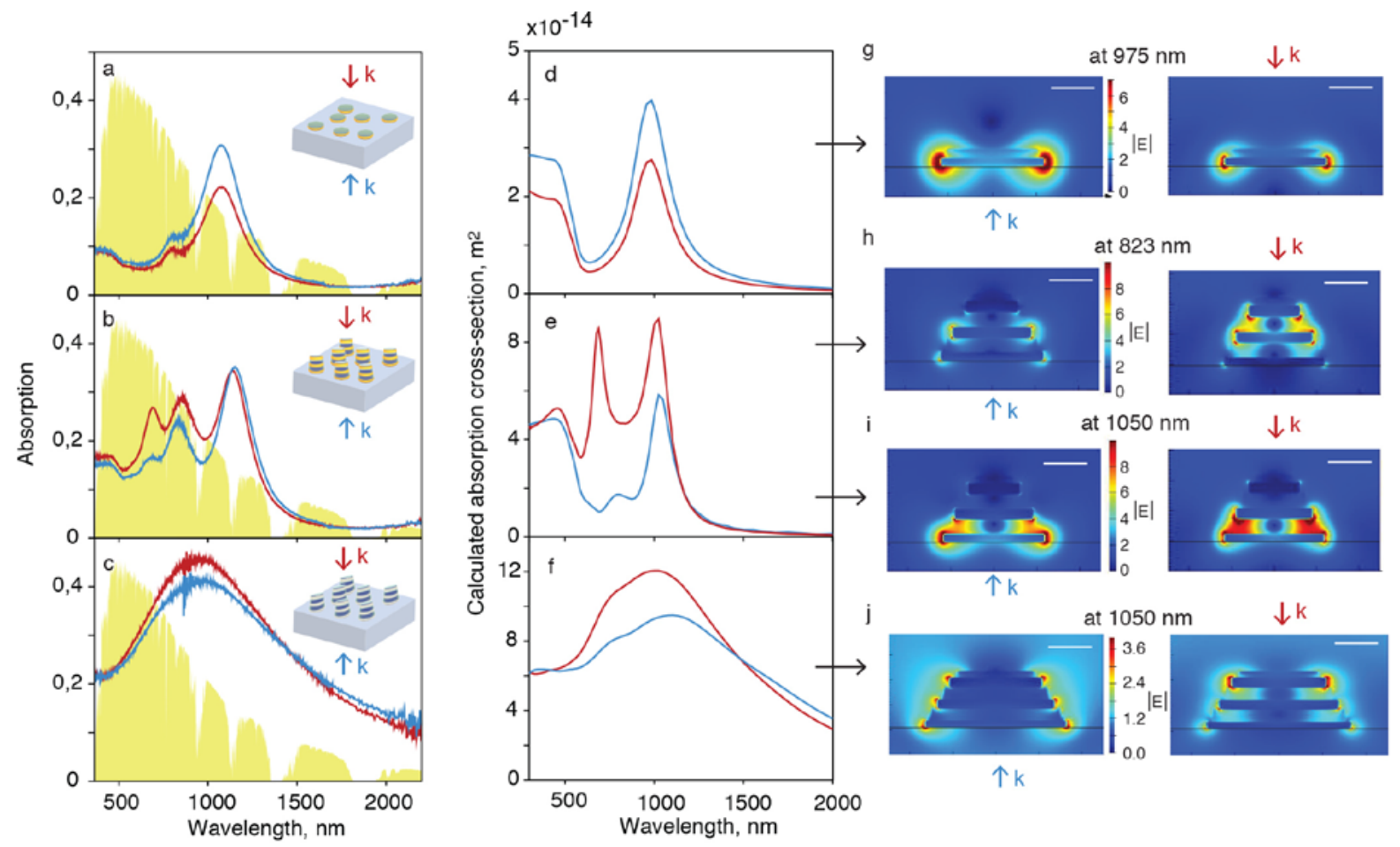

Figure 2. Experimental optical absorption spectra of (a) single-element and (b) three-element gold nanoantennas as well as (c) elliptical three-element nickel antennas. The solar spectrum is schematically shown on the background. The light incidence direction is marked in insets. (d-f) FDTD simulations of the geometries in a-c with absorption spectra and corresponding field plots of (g) single-element gold nanodisk antenna, (h-i) three-element gold nanodisks and (j) threeelement elliptical nickel nanoantennas at given wavelengths and directions of light incidence. Scale bars in (g-j) are $100 \mathrm{~nm}$.

While using gold nanodisk antennas already delivers strong directional absorption, other materials can be employed to enhance this functionality. We have previously shown that nickel nanodisks outperform gold in terms of heat generation under solar illumination. ${ }^{24}$ Nickel also provides a strong advantage in being cheap, abundant ${ }^{25}$ and sustainable material. ${ }^{26}$ Figure $2 \mathrm{c}$ shows the optical absorption of surfaces composed of elliptical three-element nickel 
nanoantennas. The absorption is higher than for the three-element gold antennas, both at the plasmonic peak position (at $950 \mathrm{~nm}$ ) and throughout the solar spectrum (shown in the background). Just as for the one-element antennas, ${ }^{24}$ the elliptical multi-element nanoantennas of this lossy metal show superior optical absorption compared to gold when integrated over the solar spectrum. The optical simulation of this system (Figure 2f) agrees well with the experimental data, including a preferential absorption upon front illumination as for the threeelement gold nanoantennas. Based on the near-field plots of Figs. 2 g-j we see that strong directional absorption is associated with stronger electromagnetic near-field enhancement in the nanoantennas. Moreover, the directionality for the elliptical three-element nickel nanoantennas is connected to concentration of the near-field primarily to the top element, while the three-element gold antennas typically show field concentration between and around two elements.

In order to correlate the optical absorption with the macroscopic heat generated by the nanoantenna surface, we employ our previously reported heat power extraction ${ }^{24}$ (see the Experimental section for details). In brief, we used a thermal camera to monitor the temperature evolution of the macroscopic sample upon solar light illumination. This directly provides the equilibrium temperature increase upon illumination, while post-processing of the dynamic light on/off cycles also enables extraction of the generated heat power density. 

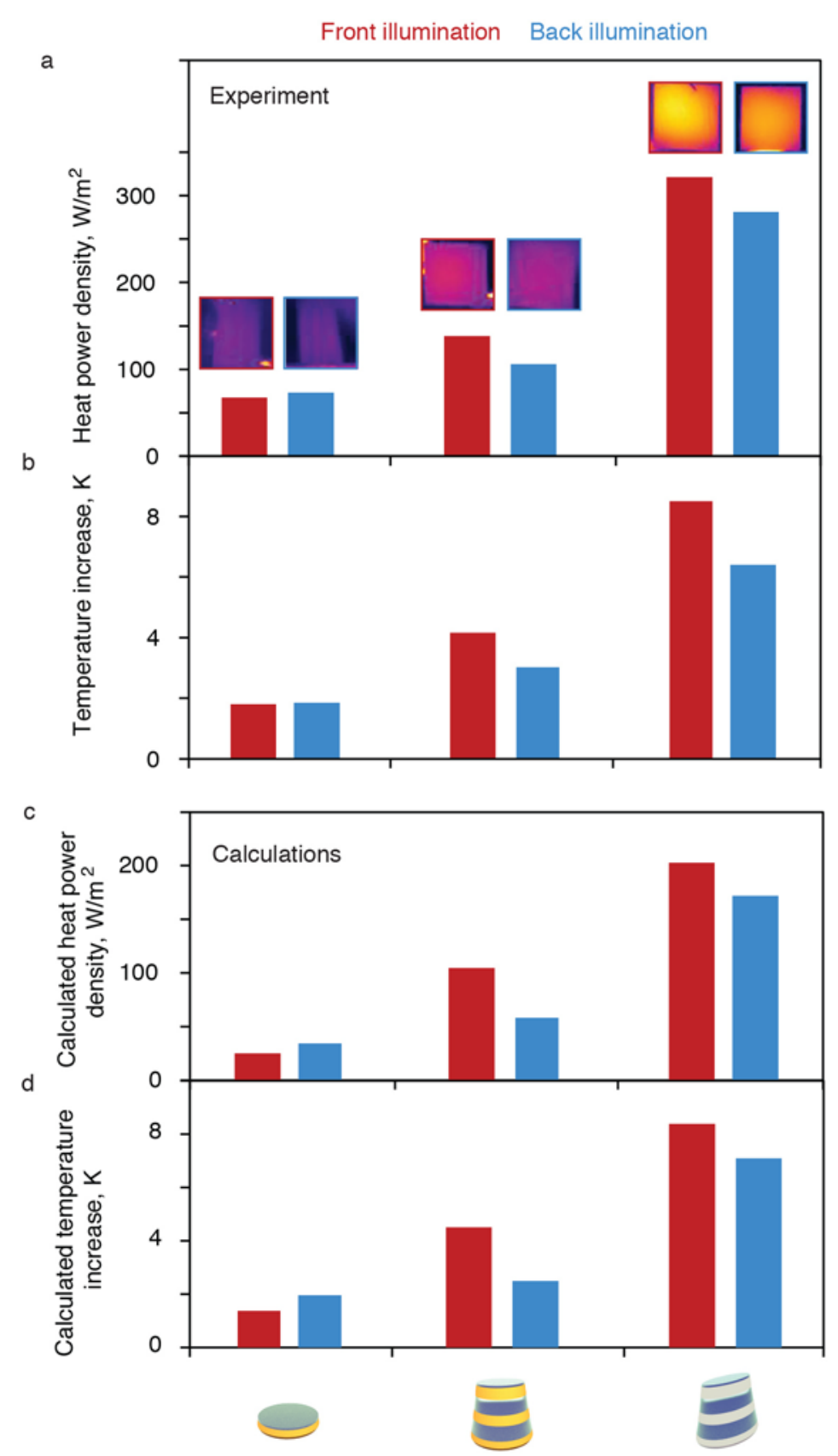

Figure 3. Experimentally determined (a) absorbed power and (b) absolute temperature increase upon solar illumination (AM1.5G) from front and backside (same samples and light directions as in Figure 2) for one- (left) and three-element (middle) gold nanoantennas and three-element nickel nanoantennas (right). (c) Simulated total solar absorption and (d) simulated temperature increase for the same systems. 
Figure 3a presents the experimentally obtained generated heat power densities of the thermoplasmonic surfaces under solar illumination (irradiance of 1 Sun, $1000 \mathrm{~W} / \mathrm{m}^{2}$, thermal measurements always done on the glass / substrate side due to the constant emissivity of the thermal photons from one measurement to another). As expected, the results follow the same trends as observed for the optical absorption, with an increase of generated heat from one- to three-element gold nanoantennas, and from gold to nickel nanoantennas while maintaining the same surface coverage. The heat generation also reproduces and confirms the trends for the directionality of light absorption discussed above.

Next, we turn to how the absorption and generated heat translates to temperature increase for the different thermoplasmonic surfaces. Equilibrium is reached when the generated heat equals the dissipated heat. In turn, dissipation arises from the temperature difference that is established between the glass and its surroundings, while the generated heat is invariant with respect to glass temperature. In the present case, heat dissipates primarily through convection and thermal radiation. The convection term can be assumed directly proportional to the temperature difference between the sample and the surrounding and the radiation term is expressed as:

$$
\varepsilon \sigma\left(T^{4}-T_{0}^{4}\right)=\frac{q}{A}
$$

where $\varepsilon$ is the emissivity of the sample, $\sigma$ is the Stefan-Boltzmann constant, $T$ and $T_{0}$ are the absolute temperatures of the sample and the surrounding, respectively, $A$ is the area of the sample and $q$ is the net radiated power. Using the following substitution:

$$
\Delta T \equiv T-T_{0}
$$

equation (1) takes the form:

$$
\varepsilon \sigma\left(\Delta T^{4}+4 \Delta T^{3} T_{0}+6 \Delta T^{2} T_{0}^{2}+4 \Delta T T_{0}^{3}\right)=\frac{q}{A}
$$


For small temperature increases from room temperature, as applicable for our case $(\Delta \mathrm{T}<10 \mathrm{~K}$ and $\mathrm{T}_{0} \approx 295 \mathrm{~K}$ ), the $4 \Delta \mathrm{TT}_{0}{ }^{3}$ term of equation (3) will dominate and the radiative losses become linear with respect to $\Delta \mathrm{T}$. We therefore expect the equilibrium temperature increase to vary approximately linearly with the absorbed power.

Figure 3b shows the measured temperature increase for the three different nanoantenna surfaces upon front (red) and back (blue) solar illumination. As expected, we observed the same trend as for the generated heat power, with higher increase in temperature for three- compared with oneelement nanoantennas and highest increase for the nickel nanoantenna surfaces. Specifically, the three-element $\mathrm{Au}$ nanoantennas surface increases its temperature by $4 \mathrm{~K}$ (front illumination) and $3 \mathrm{~K}$ (back illumination) from room temperature, which is significantly higher than for the singleelement nanoantennas showing a temperature increase of around $2 \mathrm{~K}$ for both illuminations. The elliptical three-element nickel nanoantennas produce twice the amount of heat compared to the three-element gold antennas, reaching a temperature increase of $8 \mathrm{~K}$ for front illumination. For all systems, the directionality in temperature increase agrees with the directionality of absorption and heat generation. It is instrumental to put this temperature increase into perspective. Here we draw an analogy with the molecular solar thermal fuel systems, where the controlled absorption/storage of the solar light is followed by the on-demand heat release. Such state-of-theart systems typically provide the heat release that leads to temperature increase of up to 10 degrees, ${ }^{27-28}$ i.e., directly comparable to the heat generation by our thermoplasmonic transparent surfaces.

To verify the experimental results for the temperature increase we turn again to numerical simulations (see Methods section for details). In brief, we performed the simulations in two steps. First, the FDTD method provided optical absorption spectra of the different types of 
antenna systems. These spectra were then multiplied with the model solar spectrum (AM 1.5G) and the resulting curve was spectrally integrated to extract the total absorbed power. The absorbed power then acted as the heat source in thermal simulations of the studied systems using the finite element method (FEM). The simulations comprise a nanoantenna (based on sizes and geometries from the experiments) on a glass support, with periodic boundary conditions in the plane of the thermoplasmonic surface to model an infinite array of antennas. We employed convective heat flux boundary conditions on the top and bottom of the system to simulate the heat dissipation. Figure 3c shows the calculated absorbed powers and Figure 3d shows the calculated temperature increases of the three different systems. Clearly, the trends in heat generation, temperature and directionality are all in very good agreement with the experimental results. 

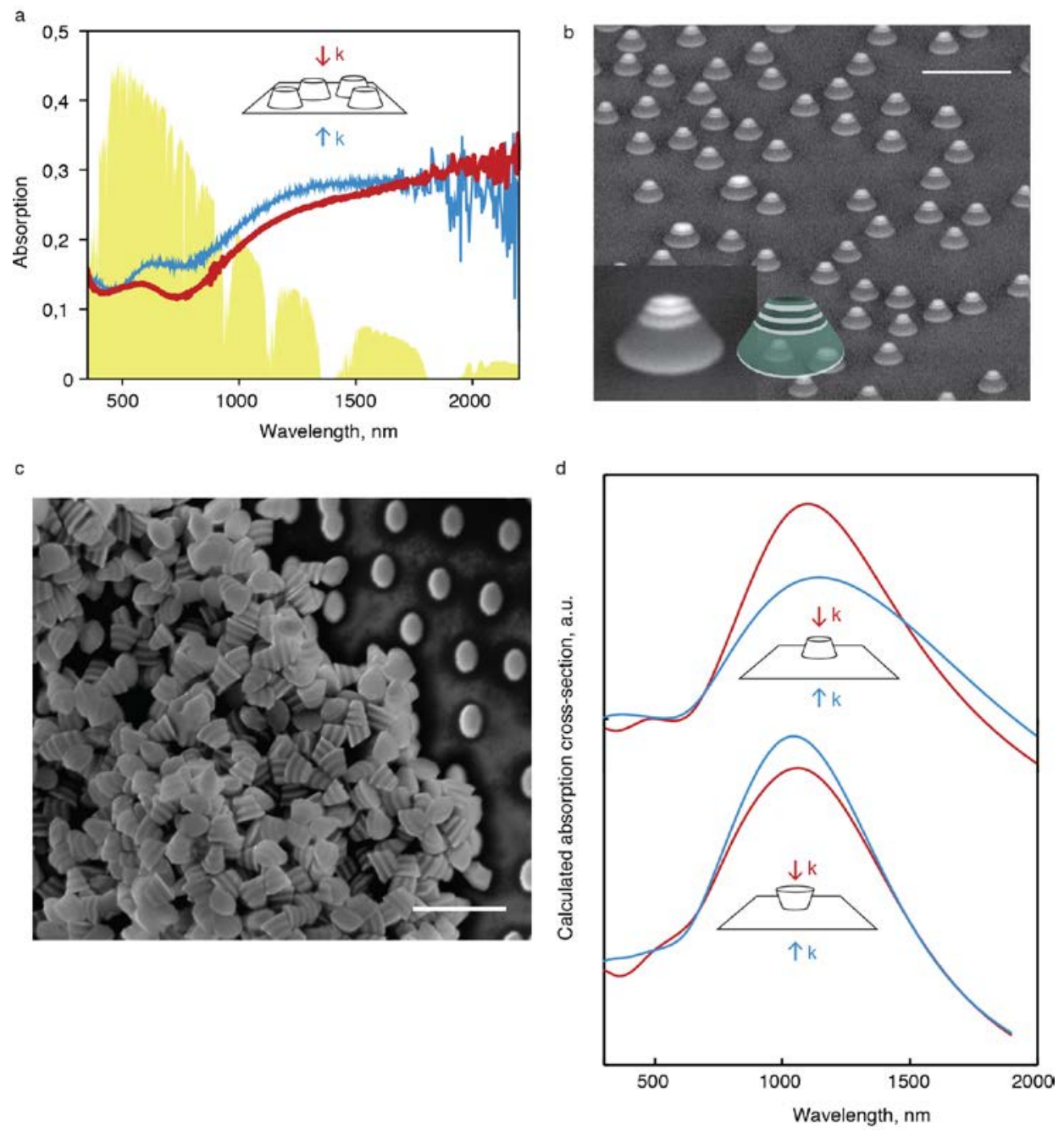

Figure 4. (a) Absorption spectra of a thermoplasmonic surface, engineered towards IR absorption and built on four-element nickel nanoantennas. Light incidence directions according to inset. (b) Actual nanoantenna geometry shown in SEM micrograph with insets of magnified single nanoantenna (left) and the schematics (right). Scale bar $2 \mu \mathrm{m}$. (c) SEM micrograph of three-element elliptical nickel nanoantennas, detached from the substrate (note the array of substrate-supported elliptical nanoantennas at the top right). Scale bar $1 \mu \mathrm{m}$. (d) Optical absorption spectra obtained from FDTD simulations for three-element nickel elliptical nanoantennas, positioned normally and upside-down on a substrate. 
While the created transparent surfaces are able to raise the equilibrium temperature of the entire macroscopic glass $(0.4 \mathrm{~mm}$ thick) by more than $8 \mathrm{~K}$ under solar illumination (1 Sun), they also need to preserve color rendering in transmission in order to be suitable for use as windows. That is, the color perception should not be distorted behind facades made by these thermoplasmonic surfaces. We use the Commission Internationale de l'Eclairage methodology ${ }^{29}$ to determine color rendering capabilities of the developed thermal surfaces. The daylight (standard illuminant D65) filtering by these surfaces (as in Figure $1 \mathrm{~b}$ ) has a very good color rendering index (CRI Ra), reaching 98.71 (standard illuminant-D, $7151 \mathrm{~K}$ ) for the elliptical three-element nickel antennas. This makes the proposed glass material truly colorless. The value can be compared with, for example, state-of-the-art luminescent solar concentrators screens that have a lower CRI $R_{a}$ of $91 .^{30}$

Approximately half of the solar energy lies outside the visible spectral region. For maximized heating with maintained visible transparency, it would be optimal to have absorption close to unity in those regions. We demonstrate the possibility to modify our elliptical three-element nickel nanoantennas towards this purpose by adding an additional nickel layer with a thick (220 nm) $\mathrm{SiO}_{2}$ dielectric spacer at the bottom of the antennas (see SEM image in Figure 4b). The absorption spectra in Figure 4a shows that these four-element nickel nanoantennas absorb considerably less in the visible region compared to the three-element nickel antennas, increasing the overall transparency while also providing a drastically increased absorption in the infrared region. In this design, shown in the inset of Figure 4b, the infrared absorption is enabled by the large bottom elliptical nickel element and the dielectric spacer upon which the three-element nickel antenna, close to the design presented in Figure 2, is placed. Optimization of the bottom- 
up process of these four-element nanoantennas would allow further enhancement of the collective absorption.

For practical large-scale manufacturing of these new transparent thermal materials, it is important that the designed geometry of the nanoantennas enables optimal heat generation for mechanically robust systems that, in the best scenario, are also structurally substrateindependent. We test this by mechanically separating the nanoantennas from the substrate by simple scratching (Figure 4c, top-view of the unaffected substrate-supported nanoantennas layer can be seen on the right). Remarkably, the three-element nanoantennas fully preserve their geometry and, according to numerical simulations shown in Figure 4d, their optical directionality. In principle, these antennas could easily be collected and re-located onto basically any surface of interest. Such mechanical robustness of the thermo-optical nanoantennas prompts for the development of various direction-selective transparent photo-thermal surfaces on substrates with challenging geometries or material compositions. A similar approach has previously been demonstrated for one-element plasmonic nanoantenna design. ${ }^{31}$

In summary, we have developed a transparent and colorless solar-powered heater surface that can be implemented on a regular window glass. The surface is built on sparse arrays of multielement gold and nickel plasmonic nanoantennas that enable directional absorption of solar light. Such directionality correlates with the generated heat at the macroscale, delivered to the entire glass substrate and allowing for temperature increases of more than $8 \mathrm{~K}$ by solar illumination under ambient conditions. The nanoantennas could also be tuned to absorb in the IR. Despite the complex composition, the nanoantennas are mechanically robust as their structural integrity is independent of the underlying substrate, opening ways of building a broad class of materials that are transparent colorless passive heater elements, powered by solar light. 


\section{EXPERIMENTAL SECTION}

\section{Sample preparation}

All samples were prepared using hole-mask colloidal lithography ${ }^{20}$. Rectangular microscope slides were used as substrates and $230 \mathrm{~nm}$ polystyrene beads were used to define the hole-masks. When fabricating the nanoelliptical antennas the hole-mask was evaporated at $45^{\circ}$ to ensure 1:1.4 ratio of short and long hole-axis. The material stacks were thermally evaporated at normal angle and consisted of $20 \mathrm{~nm} \mathrm{Au}+20 \mathrm{~nm} \mathrm{SiO}$ for single element antennas, and $20 \mathrm{~nm} \mathrm{Au}+40 \mathrm{~nm}$ $\mathrm{SiO}_{2}+28 \mathrm{~nm} \mathrm{Au}+40 \mathrm{~nm} \mathrm{SiO}_{2}+30 \mathrm{~nm} \mathrm{Au}+20 \mathrm{SiO}_{2}$ for triple element antennas. The diameter of the stack decreases with the height due to the orifices of the mask being gradually closed by the deposited material. For elliptical nanoantennas the same layer thicknesses were used, but Au was replaced by $\mathrm{Ni}$. Also, $\mathrm{SiO}_{2}$ was replaced by $\mathrm{Al}_{2} \mathrm{O}_{3}$ for fabrication technical reasons. The elliptical four-element nickel nanoantennas for enhanced absorption of infrared radiation was fabricated using an elliptical mask made using $510 \mathrm{~nm}$ diameter polystyrene beads, with the same aspect ratio as for the $230 \mathrm{~nm}$ mask. The bottom Ni layer was $20 \mathrm{~nm}$ thick and the dielectric spacer was 220 nm thick. $\mathrm{SiO}_{2}$ was used as spacer layer in this case (also for fabrication technical reasons).

\section{Spectroscopic absorption measurement}

Optical absorption spectra were recorded in a Cary 5000 spectrophotometer from Varian (now Agilent technologies, Inc., Santa Clara, CA) equipped with an integrating sphere of model DRA 2500, Varian. The samples were mounted with a center mount sample holder and measurements were done in "transflectance" mode, meaning that both transmitted and reflected light was collected by the detector. The angle of the incident light was kept at $9^{\circ}$ off-normal in order to 
include the specularly reflected beam, which would otherwise escape undetected through the beam entrance port of the sphere. In the case of nanoelliptical antennas, the spectroscopic measurements are made separately with light polarized parallel to each axis. The absorption data then are the mean of the two perpendicular measurements. This was necessary because the light in the Cary 5000 spectrometer is highly polarized, while the solar simulator, naturally, produces completely unpolarised light.

\section{Thermally measured absorption}

Thermal absorption measurements were carried out in a similar way as previously reported ${ }^{24}$. The difference being that measurements were also made with the light source placed on the opposite side of the samples as seen from the thermal camera. A ss150 solar simulator from Sciencetech, inc., London, Ontario, was used as a light source and an A645 thermal camera from FLIR systems inc, Wilsonville, Oregon, was used to record the thermal images of the substrate. The software Flir ResearchIR 3.4, FLIR systems, was used to extract the temperature.

Quantification of the absorbed power was done by first multiplying the temperature increase of the sample by its specific heat $\left(\mathrm{Cv}=820 \mathrm{~J} /(\mathrm{K} \cdot \mathrm{kg})\right.$, density $\left(\rho=2510 \mathrm{~kg} / \mathrm{m}^{3}\right)$ and thickness $(\mathrm{d}=$ $4 \cdot 10^{-4} \mathrm{~m}$ ) to convert the trace into a quantity with dimension $\mathrm{J} / \mathrm{m}^{2}$ which has the physical meaning of net added thermal energy per unit surface. Subsequent differentiation of this quantity with respect to time gives the net power that infers this change of thermal energy per unit area. Re-plotting the data sets against the sample temperature instead of time reveals two traces corresponding to the light on-phase and light off-phase respectively. Making two linear curve fits to these traces gives two almost parallel lines. The separation between these lines represents the heating power from the absorption of the AM1.5G spectrum. The value of the temperature axis at which the light-on line equals zero is the equilibrium temperature upon illumination. 


\section{Optical numerical simulations}

We performed finite-difference time-domain (FDTD) simulations (absorbed power, electric nearfield intensity, far-field spectra) on the thermoplasmonic surfaces by using the commercial software Lumerical FDTD Solutions (http://www.lumerical.com/fdtd.php). To evaluate the absorption of the nanoelliptical antennas, the same strategy as in the experiment was applied. I.e., the absorption is calculated with light, polarized along the two nanoellipse axes, and the resulting 'unpolarized' light absorption is the mean of the two data. Nanoantennas are modelled with dimensions from the experiment and are placed on $\mathrm{SiO}_{2}$ substrate. Uniform calculational mesh is $2.5 \mathrm{~nm}$.

\section{Thermal simulations}

We carried out finite element method (FEM) heat transfer simulations using the COMSOLMultiphysics software package (version 5.1). The geometry of the plasmonic structures was the same as used on the optical simulations with a $0.4 \mathrm{~mm}$ glass as substrate. The size of the simulation was $650 \mathrm{~nm}$ on $\mathrm{x}$ and $\mathrm{y}$, with periodic boundary conditions to simulate an infinite array. A convective heat flux was used on $\mathrm{z}$ (top and bottom of the simulation) with a heat transfer coefficient of $10 \mathrm{~W} /(\mathrm{m} 2 \cdot \mathrm{K})$, a common value used for air. We incorporated the total absorbed power by the nanostructures obtained in the optical simulations as heat sources. We used the material properties of gold and glass provided by the COMSOL-Multiphysics software package.

\section{AUTHOR INFORMATION}

\section{Corresponding Author}

*E-mail: alexd@physics.gu.se 


\section{Author Contributions}

The manuscript was written through contributions of all authors. All authors have given approval to the final version of the manuscript.

\section{Notes}

The authors declare no competing financial interests.

\section{ACKNOWLEDGMENT}

This work is supported by the Swedish Foundation for Strategic Research (SSF) through RMA08-0109. AD acknowledges the Swedish Research Council (VR). VM acknowledges the support from EU FP7 project "PRIMA" - 248154 and EU FP7 project 'PhotoNVoltaics' 309127. DT and MPJ acknowledge financial support from the Wenner-Gren Foundations, the Swedish Research Council, the Swedish Foundation for Strategic Research, the Royal Swedish Academy of Sciences, the ÅForsk Foundation, and the Swedish Government Strategic Research Area in Materials Science on Functional Materials at Linköping University (Faculty Grant SFOMat-LiU No. 2009 00971). We acknowledge Irina Zubritskaya for the SEM images. 


\section{REFERENCES}

(1) Neumann, O.; Urban, A. S.; Day, J.; Lal, S.; Nordlander, P.; Halas, N. J., ACS Nano 2012, 7, 42-49.

(2) Lennartson, A.; Roffey, A.; Moth-Poulsen, K., Tetrahedron Lett. 2015, 56, 1457-1465.

(3) Scharf, H.-D.; Fleischhauer, J.; Leismann, H.; Ressler, I.; Schleker, W.-g.; Weitz, R., Angew. Chem. Int. Ed. Engl. 1979, 18, 652-662.

(4) Höglund, I.; Pettersson, O.; Åhlgren, B. Fönsterteknik. Byggförl.: Stockholm, 1984; 272 S.

(5) Lechner, N. Heating, cooling, lighting : design methods for architects. 2nd ed.; Wiley: New York, 2001; p xvii, 620 p.

(6) Brongersma, M. L.; Halas, N. J.; Nordlander, P., Nature Nanotech. 2015, 10, 25-34.

(7) Mubeen, S.; Lee, J.; Singh, N.; Kramer, S.; Stucky, G. D.; Moskovits, M., Nature Nanotech. 2013, 8, 247-251.

(8) Niesen, B.; Rand, B. P.; Van Dorpe, P.; Cheyns, D.; Tong, L.; Dmitriev, A.; Heremans, P., Adv. Energy Mater. 2013, 3, 145-150.

(9) Mukherjee, S.; Zhou, L.; Goodman, A. M.; Large, N.; Ayala-Orozco, C.; Zhang, Y.; Nordlander, P.; Halas, N. J., J. Am. Chem. Soc. 2014, 136, 64-67.

(10) Atwater, H. A.; Polman, A., Nature Mater. 2010, 9, 205-213.

(11) Donner, J. S.; Morales-Dalmau, J.; Alda, I.; Marty, R.; Quidant, R., ACS Photon. 2015, $2,355-360$.

(12) Donner, J. S.; Baffou, G.; McCloskey, D.; Quidant, R., ACS Nano 2011, 5, 5457-5462.

(13) Baffou, G.; Quidant, R., Laser Photon. Rev. 2013, 7, 171-187.

(14) Tordera, D.; Zhao, D.; Volkov, A. V.; Crispin, X.; Jonsson, M. P., Nano Lett. 2017, 17, 3145-3151.

(15) Maier, S. A. Plasmonics : fundamentals and applications. Springer: New York, 2007; p xxiv, 223 p.

(16) Wadell, C.; Antosiewicz, T. J.; Langhammer, C., Nano Lett. 2012, 12, 4784-4790.

(17) Shegai, T.; Chen, S.; Miljkovic, V. D.; Zengin, G.; Johansson, P.; Kall, M., Nat.

Commun. 2011, 2:481.

(18) Langhammer, C.; Kasemo, B.; Zoric, I., J. Chem. Phys. 2007, 126, 267203.

(19) Moreau, A.; Ciraci, C.; Mock, J. J.; Hill, R. T.; Wang, Q.; Wiley, B. J.; Chilkoti, A.;

Smith, D. R., Nature 2012, 492, 86-89.

(20) Fredriksson, H.; Alaverdyan, Y.; Dmitriev, A.; Langhammer, C.; Sutherland, D. S.;

Zaech, M.; Kasemo, B., Adv. Mater. 2007, 19, 4297-4302.

(21) Tong, L.; Pakizeh, T.; Feuz, L.; Dmitriev, A., Sci. Rep. 2013, 3:2311.

(22) Dmitriev, A.; Hagglund, C.; Chen, S.; Fredriksson, H.; Pakizeh, T.; Kall, M.; Sutherland, D. S., Nano Lett. 2008, 8, 3893-3898.

(23) Verellen, N.; Van Dorpe, P.; Huang, C.; Lodewijks, K.; Vandenbosch, G. A. E.; Lagae, L.; Moshchalkov, V. V., Nano Lett. 2011, 11, 391-397.

(24) Jonsson, G. E.; Miljkovic, V.; Dmitriev, A., Sci. Rep. 2014, 4:5111.

(25) Hans Wedepohl, K., Geochim. Cosmochim. Acta 1995, 59, 1217-1232.

(26) Denkhaus, E.; Salnikow, K., Crit. Rev. Oncol. Hematol. 2002, 42, 35-56.

(27) Zhitomirsky, D.; Cho, E.; Grossman, J. C., Adv. Energy Mater. 2016, 6, 1502006-n/a.

(28) Moth-Poulsen, K.; Coso, D.; Borjesson, K.; Vinokurov, N.; Meier, S. K.; Majumdar, A.; Vollhardt, K. P. C.; Segalman, R. A., Energy Environ. Sci. 2012, 5, 8534-8537. 
(29) Münch, W. CIE 13.3-1995 Method of Measuring and Specifying Colour Rendering Properties of Light Sources. Commission Internationale de l'Eclairage: Vienna, 1995.

(30) Meinardi, F.; McDaniel, H.; Carulli, F.; Colombo, A.; Velizhanin, K. A.; Makarov, N. S.; Simonutti, R.; Klimov, V. I.; Brovelli, S., Nature Nanotech. 2015, 10, 878-885.

(31) Mirin, N. A.; Halas, N. J., Nano Lett. 2009, 9, 1255-1259. 
TOC graphics

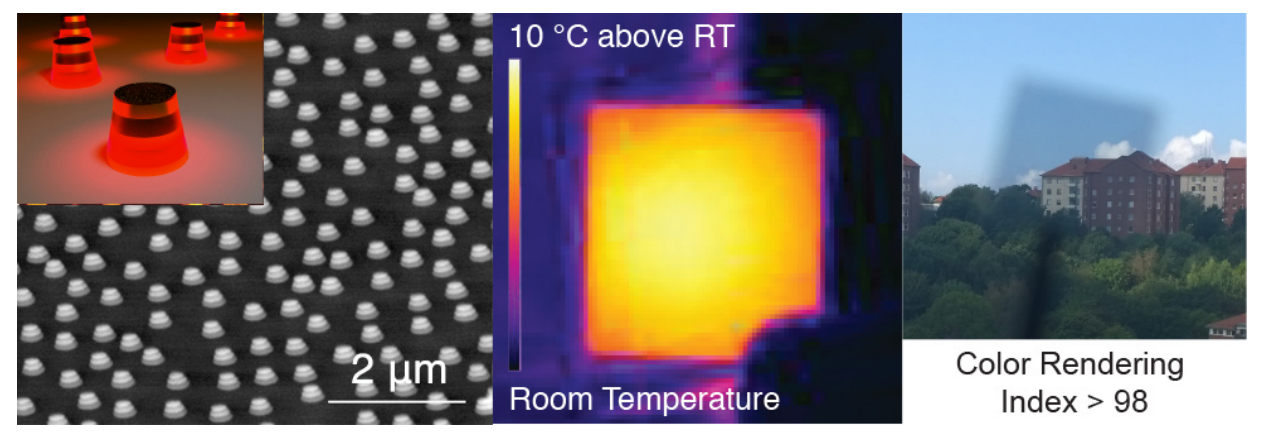

